\title{
Acesso Venoso pela Via Intra-Óssea em Urgências Médicas*
}

\author{
Venous Access by Intraosseous Access in Medical Urgencies
}

John Cook Lane1, Hélio Penna Guimarães ${ }^{2,3}$

\section{RESUMO}

JUSTIFICATIVA E OBJETIVOS: Inicialmente introduzida em 1922, a técnica de acesso intra-ósseo foi extensamente utilizada até os anos de 1940 e revisada nos anos 1980. Considerando sua recomendação em diretrizes de reanimação cardiopulmonar, o objetivo deste estudo foi apresentar uma revisão histórica e principais aspectos clínicos sobre acessos intra-ósseos ao sistema venoso.

CONTEÚDO: Foi realizada uma busca por artigos originais através da palavra-chave "acesso intra-ósseo" na base de artigos MedLine (1950 a janeiro de 2008). Adicionalmente, referências destes artigos, capítulos de livros e artigos históricos foram avaliados para esta revisão. Portais na internet de fabricantes de equipamentos para punção intra-óssea também foram consultados. Foram identificados e revisados 231 artigos, e o presente estudo condensou os principais resultados descritos. Foram considerados ensaios clínicos na língua inglesa, estudos retrospectivos e artigos de revisão.

1. Professor Titular Colaborador da Faculdade de Ciências Médicas da Universidade Estadual de Campinas (UNICAMP). Médico da Fundação Centro Médico de Campinas.

2. Médico Diarista da UTI da Disciplina de Clínica Médica da Universidade Federal de São Paulo (UNIFESP-EPM). Coordenador do Centro de Treinamento em Emergências do Instituto Dante Pazzanese de Cardiologia e Centro de Treinamento em Medicina de Urgência da Sociedade Brasileira de Clínica Médica-SBCM.

3. Título de Especialista em Medicina Intensiva pela AMIB-AMB.

*Recebido da Fundação Centro Médico de Campinas, SP; Centro de Treinamento em Emergência do Instituto Dante Pazzanese de Cardiologia, São Paulo, SP.

Apresentado em 23 de dezembro de 2007

Aceito para publicação em 06 de fevereiro de 2008

Endereço para correspondência:

Prof. Dr. John Cook Lane

Rua Cajá, 230 - AlphaVille

13098.334 Campinas, SP

E-mail : vanlane@uol.com.br

(C)Associação de Medicina Intensiva Brasileira, 2008
CONCLUSÕES: O acesso intra-ósseo é usado principalmente para garantir rapidamente um acesso venoso quando há algum retardo ou dificuldade para acesso venoso periférico. Consiste em técnica de simples aprendizado e suas complicações são inferiores a $1 \%$. A maior parte dos fármacos utilizados em emergências pode ser administrada por esta via em suas doses rotineiras. $\mathrm{O}$ acesso intra-ósseo pode ser usado com segurança em diferentes locais de punção, tanto em adultos quanto crianças. Este acesso pode ser satisfatoriamente utilizado para coleta sanguínea objetivando análise de gases e bioquímica sanguínea, infusão de sangue e hemoderivados, solução fisiológica para reposição volêmica em estados de choque, parada cardiorrespiratória e outras emergências, quando o acesso venoso convencional não pode ser rapidamente efetivado.

Unitermos: acesso intra-ósseo, acesso venoso

\section{SUMMARY}

BACKGROUND AND OBJECTIVES: First introduced in 1922, the intraosseous access technique was extensively used in the 1940's and revised in the 1980's. Since this technique is recommended in actual cardiopulmonary resuscitation guidelines, the authors present an historical and clinical review of intraosseous access to the venous system.

CONTENTS: The MedLine (1950 to January 2008) database was searched for pertinent abstracts, using the key term intraosseous access. Additional references and historical papers were obtained from the bibliographies of the articles reviewed. Manufacturer Web sites were used to obtain information about intraosseous venous $(\mathrm{IO})$ insertion devices. Were identified and reviewed 231 articles, and this present article condensed the mainly the principal findings described. All available English-language clinical trials, retrospective studies and review articles describing $\mathrm{IO}$ drug administration were reviewed.

CONCLUSIONS: The intraosseous access is used mainly to gain rapid access to the intravenous system 
when there is delay in obtaining the latter one. The technique is simple to learn. The complications rate is less than $1 \%$. Most emergency drugs can be administered in the same doses used by intravenous routes. Bone access can be used in children and adults of any age in several sites. This access can be used satisfactorily to draw blood for cross-matching, blood gases and blood chemistries and emergency infusion of blood and its derivatives, saline solutions for volume replacement in shock, cardiac arrest and emergencies when an intravenous access cannot be made readily available.

Key Words: Intraosseous access, Intravenous access.

\section{HISTÓRICO}

A via intra-óssea (IO) como via de acesso à circulação venosa foi descrita em inicialmente, em 1922, por Drinker e col. ${ }^{1}$. Em 1934, Josefson ${ }^{2}$ publicou um estudo sobre a via IO, como substituto emergencial para a administração de líquidos em crianças. A técnica passou a ser usada com freqüência crescente, entrando na rotina pré-hospitalar e pronto-socorros na década de $1940^{3-10}$. Com o surgimento dos cateteres introduzidos sob agulhas para acesso venoso, esta via caiu em desuso até a década de 1980, quando passou a ser reaplicada em crianças ${ }^{11-25}$ e recentemente em adultos ${ }^{19-21}$. O acesso intra-ósseo voltou a ganhar evidência com as atuais diretrizes mundiais de reanimação cardiopulmonar, que o posicionam como a segunda opção em seqüência de acessos ou vias de administração de medicamentos, no caso de insucesso na obtenção de um acesso venoso periférico ${ }^{19,22-24}$.

\section{INTRODUÇÃO}

A obtenção de uma via venosa em crianças e adultos em estado crítico, tem como alternativa a via 10 , quando a primeira não for estabelecida num curto prazo ou após três tentativas ${ }^{16,19,22-24}$.

Nos ossos longos, os sinusóides da medula óssea drenam para canais venosos que levam o sangue ao sistema venoso; a vantagem anatômica da medula óssea é seu funcionamento como uma veia rígida que não colaba em estado de hipovolemia e no choque circulatório periférico ${ }^{16}$. Assim, a via 10 tem sido usada com eficácia como via de emergência na parada cardiorrespiratória, nos choques hipovolêmico e séptico, queimaduras graves, estados epiléticos prolongados e desidratação intensa, principalmente em crianças ${ }^{16}$.

\section{TÉCNICA}

A punção em crianças pode ser realizada com uma agulha comum de injeção tamanho $25 \times 12$, agulha para raquianestesia ou com trepano para biópsia de medula óssea.

O local para punção mais freqüentemente usado em crianças corresponde à face interna da tíbia, área anatômica e recoberta apenas por pele, pouco tecido celular subcutâneo e periósteo quando em cerca de 1 a $3 \mathrm{~cm}$ abaixo da tuberosidade tibial. A agulha deve ser direcionada levemente inclinada $\left(15\right.$ a $\left.30^{\circ}\right)$ para a parte distal evitando a punção da cartilagem de crescimento metafisária. Ao se sentir a ponta da agulha atravessando a córtex óssea, não mais se deve aprofundá-la.

A penetração da agulha na cavidade medular pode ser determinada pelos seguintes parâmetros:

1) Perda discreta da resistência óssea;

2) A agulha permanece na posição "de pé" sem suporte;

3) Pode-se aspirar medula óssea (semelhante a sangue);

4) A infusão de bolus de 5 a $10 \mathrm{~mL}$ de solução fisiológica com uma seringa, resulta em mínima resistência e não deve haver evidência de infiltração no subcutâneo;

Se o fluxo da solução é facilmente administrado e não há extravasamento, deve-se conectar o equipo de soro e afixá-lo à coxa como demonstrado na figura 1 . A agulha deve ser protegida de abalos, circundando-a com gaze e esparadrapo.

O procedimento costuma ser de fácil execução sendo que, em média, o número de tentativas para sua execução com sucesso, em crianças, costuma ser de 1,6 tentativa de punção, com tempo médio para a obtenção de até oito minutos ${ }^{26}$.

Em pacientes adultos, utilizando alguns equipamentos mais modernos para punção (Figura 2), este acesso pode ser garantido em até 57-127 segundos, cerca de 2 minutos, com sucesso, quando realizado por paramédicos habilitados, em treinamento mínimo de cerca de $90 \mathrm{~min}^{27}$. Com agulhas convencionais para punção óssea, este tempo em geral é de 4,4 $\pm 2,8 \mathrm{~min}^{28}$. O acesso intra-ósseo obtido em situações de emergência por ser mantido, em geral, até 24 horas do início de sua inserção, havendo necessidade de substituí-lo após este período, não apenas pela progressiva perda de sua eficiência como também por riscos adicionais de osteomielite e embolia gordurosa ${ }^{22,24}$. 


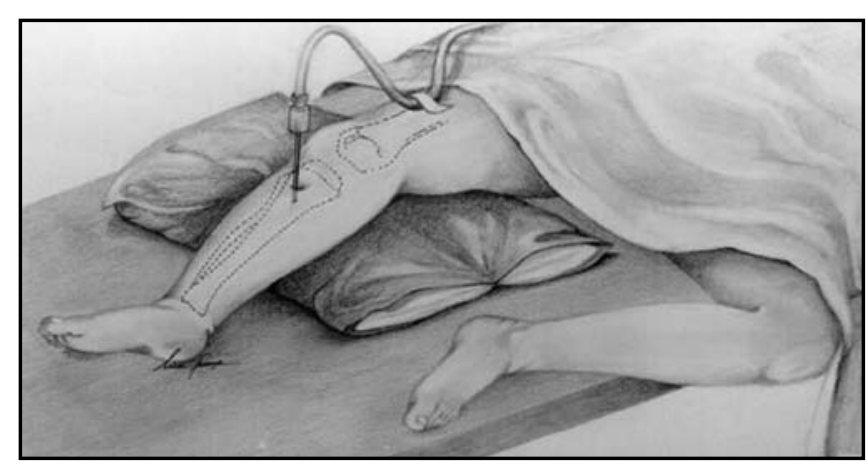

Figura 1 - Punção Intra-Óssea em Crianças.

Um coxim debaixo do joelho com leve rotação externa coxofemoral facilita a estabilidade da extremidade. (ilustração cedida gentilmente por Dr. Fábio Hüsemann Menezes, modificada de Menezes FH - Acesso à Circulação Venosa, em: Lane JC, Albarran-Sotelo R - Reanimação Cardiorrespiratória Cerebral. Rio de Janeiro: Editora Medsi, 1993;177).

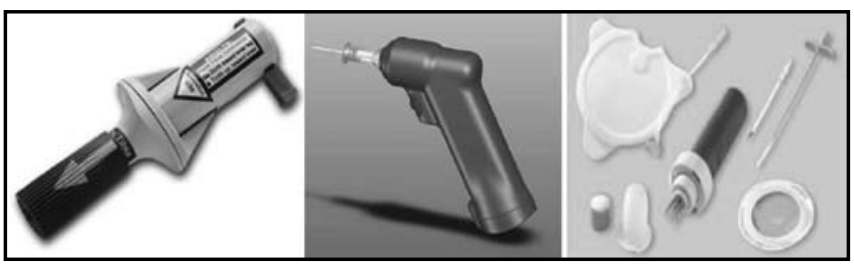

Figura 2 - Equipamentos para Punção Intra-Óssea em Adultos B.I.G. ${ }^{\circledR}$ EZ10 ${ }^{\circledR}$ FAST $1^{\circledR}$

(fonte: Arquivo pessoal dos autores e www.waismed.com)

A tabela 1 mostra outros locais da infusão por via 10 Descritos na Literatura Médica

Tabela 1 - Locais de Infusão Intra-Óssea

\begin{tabular}{|c|c|}
\hline Locais & Referências \\
\hline Tíbia & Adultos $^{19}$ e crianças $^{20}$ \\
\hline Maléolo medial & Adultos $^{19}$ \\
\hline Esterno & Adultos ${ }^{21,25}$ \\
\hline Crista ilíaca & Adultos 25,29 \\
\hline Clavícula & Adultos $^{29}$ \\
\hline Fêmur & Crianças $^{15,31}$ \\
\hline Úmero & Crianças $^{32}$ \\
\hline Calcâneo & Crianças $^{20,33}$ \\
\hline
\end{tabular}

\section{COMPLICAÇÕES}

As complicações da infusão por via IO são infreqüentes, sendo a mais comum a infusão de fluídos no subcutâneo e, mais raramente, subperiostal. Poderá ocorrer extravasamento de líquidos pelo local de punção e ou a formação de coágulo na agulha ${ }^{15}$. A necrose tecidual e, mais raramente, a síndrome compartimental podem ocorrer relacionadas à inserção inadequada da agulha e/ou o seu deslocamento para outra estrutura ${ }^{22,24}$.

A celulite localizada ou abscesso subcutâneo tem sido relatado em 0,7\% de casos (cinco entre 694) ${ }^{15}$. Existe potencialmente o risco de osteomielite, sendo que Rosetti e col. ${ }^{11}$, descreveram somente 27 casos em 4270 infusões por via $1 \mathrm{O}$ avaliadas $(0,6 \%)$ na maior série avaliada de casos.

A preocupação do efeito tardio da $\mathrm{IO}$ na placa de crescimento ósseo também se fez presente com a intensificação do uso da técnica em crianças; entretanto, este efeito adverso não foi demonstrado em inúmeros estudos clínicos e experimentais ${ }^{15}$. Também a possibilidade de embolia gordurosa, ainda que possível não tem sido relatada com freqüência, particularmente considerando que a medula de crianças que é quase livre de gorduras ${ }^{15}$.

Poucos óbitos têm sido atribuídos ao procedimento e todos foram relacionados com punção esternal em crianças; esses casos foram relacionados à punção esternal em crianças (abaixo de três anos de idade) que resultaram em mediastinite, hidrotórax ou ferimentos do coração ou grandes vasos ${ }^{15}$.

Finalmente a dor tem sido relacionada ao aumento da pressão intramedular, o que não é problema com infusões mais lentas ou em pacientes inconscientes ${ }^{34,35}$.

\section{FÁRMACOS E FLUÍDOS ADMINISTRADOS POR VIA INTRA-ÓSSEA}

Inúmeros fluídos e fármacos têm sido administrados por via IO. Dubick e Holcomb ${ }^{36}$ revisaram, em 2000, cerca de 100 referências analisando os fluídos e suas velocidades de infusão, assim como também os fármacos administrados. Os fluídos mais usados foram solução fisiológica, sangue, plasma, solução de Ringer, $\mathrm{NaCl} /$ dextran e glicose hipertônica. Não apenas neste artigo, mas também em outras distintas referências, fármacos utilizados através deste acesso incluem epinefrina, dopamina, dobutamina, atropina, adenosina, digoxina, corticosteróides, morfina e diazepínicos ${ }^{35-40}$. As doses da via $I O$ são a mesmas usadas por via veno$\mathrm{sa}^{33}$. Neste cenário, no entanto, discute-se a real efetividade da absorção dos fármacos e quais parâmetros adequados para tal avaliação. Von Hoff e col. ${ }^{38}$, comparam a farmacocinética do sulfato de morfina administrado por via intra-óssea versus via venosa, em estudo prospectivo, aleatório em grupos para receber 5 mg em bolus de sulfato de morfina por via intra-óssea ou venosa seguida da administração rotineira em $24 \mathrm{~h}$. Amostras sanguíneas seriadas de $5 \mathrm{~mL}$ eram avaliadas em até 8 hos infusão por radioimunoensaio e determinava-se a concentração plasmática máxima (Cmax), o tempo para atingir concentração plasmática máxima 
(TCmax) e a curva tempo-concentração plasmática (AUC). Houve o claro achado de diferença na Cmax (235 \pm 107 versus $289 \pm 197 \mathrm{ng} / \mathrm{mL}$, por via IO versus venosa, respectivamente), Tmax (1,3 0 0,5 versus $1,4 \pm$ 0,5 minuto) e AUC (0-infinity) (4372 \pm 1785 versus 4410 $\left.\pm 1930 \mathrm{ng} \mathrm{min}^{-1} \cdot \mathrm{mL}^{-1}\right)$. Estes resultados suportaram a hipótese de bioequivalência desta via para administração medicamentosa e descrevem o método adequado para avaliação de aspectos farmacológicos de medicamentos administrados por esta via.

\section{DISCUSSÃO}

Durante as últimas décadas, foi acumulado na literatura um volume expressivo de estudos sobre o uso da via IO. Apesar do uso disseminado de cateteres venosos, e reconhecido o potencial de limitações de uso em emergências na esfera pré-hospitalar, incluindo demora e ou inviabilidade de obtenção de via venosa, presença de veias colabadas, coagulação no local de infusão e a presença de obesidade ${ }^{36}$. Recentemente a via IO foi também introduzida mais largamente por Dubick e Holcomb ${ }^{36}$ também na área militar, incluindo nas guerras no Oriente Médio em 2002.

Waismann e col. ${ }^{41}$ fizeram uma revisão do uso da via $I O$ no trauma e descreveram nas crianças, como a técnica mais versátil, a colocação da via IO na parte proximal da tíbia. Considerando, no entanto, que a medula vermelha nos ossos longos é paulatinamente substituída por medula gordurosa, a partir de cinco anos, a tíbia teria a vantagem de acesso 10 , por ter uma medula mais ampla, apesar de seu córtex ósseo mais duro, que possivelmente torna difícil a colocação da agulha manualmente no adulto.

Com o advento de novos equipamentos e bombas injetoras a agilidade do acesso ósseo nos ossos longos dos adultos foi otimizada e trouxe sua prática mais próxima à beira-do-leito em adultos ${ }^{39-44}$.

O osso esterno ${ }^{46-48}$ também se caracteriza como local atrativo para a infusão 10 em adultos, por ser macio, com bom espaço medular, gasometria uniforme e recoberta por uma camada fina de pele. Esta via não deve ser usada em crianças abaixo da idade de três anos, devido ao risco de a agulha lesar o coração ou os grandes vasos.

Além da infusão de líquidos e fármacos, a via 10 tem sido usada para avaliação bioquímica sanguínea ${ }^{49-51}$, avaliação do equilíbrio ácido-básico, quantificação da hemoglobina e sua tipagem, ainda que em estados de hipotermia ${ }^{50}$. Estes exames devem ser colhidos antes da infusão de líquidos.

Por fim, Waisman e col. ${ }^{39}$ usaram a técnica $1 \mathrm{O}$ para obter anestesia regional em 109 cirurgias ortopédicas nas extremidades superiores e inferiores; destes, a anestesia foi satisfatória em 106 pacientes e, em três, houve ineficiência por erros técnicos na punção e infusão. O calcâneo ${ }^{39,41,45,47,51,52}$, clavícula ${ }^{42-44}$, esta última com maior freqüência, têm sido usadas também como vias alternativas.

\section{CONLUSÃO}

O acesso venoso pela via intra-óssea é seguro, efetivo para reposição volêmica, administração de fármacos e exames laboratoriais em todas as idades. A via intraóssea deve ser obtida quando o acesso venoso não for rapidamente estabelecido. Esta via é indicada inclusive em pacientes em choque e parada cardiorrespiratória, sendo a única exceção nos recém-nascidos, quando o acesso pela veia umbilical deve ser preferencial.

\section{REFERÊNCIAS}

01. Drinker CK, Drinker KR, Lund CC - The circulation in the mammalian bone marrow. Am J Physiol, 1922;62:1-92.

02. Josefson A - A new method of treatment intraossal injections. Acta Med Scand, 1934;81-550-564.

03. Tocantins LM - Rapid absorption of substances injected in the bone marrow. Proc Doc Exp Biol Med, 1940;45:292-296.

04. Tocantins LM, O'Neill JF, Jones HW - Infusions of blood and other fluids via the bone marrow applications in pediatrics. JAMA, 1941;117:1229-1234.

05. Papper EM - The bone marrow route for injecting fluids and drugs in to the general circulation. Anesthesiology, 1942;3:307-313.

06. Meola F - Bone marrow infusions as routine procedure in children. $J$ Pediatr, 1944;25:13:15-19.

07. Tocantins LM, O'neill JF - Complications in intra-osseous therapy. Ann Surg, 1945;122:266-277.

08. Heinied S, Sondergaard T, Tudvad F - Bone marrow infusion in childhood experiences from a thousand infusions. J Pediatr, 1947;30:400-412.

09. Elston JT, Jaynes RV, Kaump DH et al - Intraosseous infusions in infants. Am J Clin Pathol, 1947;17:143-150.

10. Massey LW - Bone-marrow infusions: intratibial and intravenous routes compared. Br Med J, 1950;22:(4672):197-198.

11. Berg RA - Emergency infusion of catecholamines into bone marrow. Am J Dis Child, 1984;138:810-811.

12. Rosetti VA, Thompson BM, Miller $\mathrm{J}$ et al - Intraosseous infusion: an alternative route of pediatric intravascular access. Ann Emerg Med, 1985;14:885-888.

13. Glaeser PW, Losek JD - Emergency intraosseous infusions in children. Am J Emerg Med, 1986;4:34-36.

14. Spivey WH - Intraosseous infusions. J Pediatr, 1987;111:639-643.

15. Mofenson HC, Tascone A, Caraccio TR - Guidelines for intraosseous infusions. J Emerg Med, 1988;6:143-146.

16. Smith RJ, Keseg DP, Manley LK et al - Intraosseous infusions by prehospital personnel in critically ill pediatrics patients. Ann Emerg Med, 1988;17:491-495.

17. Wayne MA - Intraosseous vascular access. Devices, sites \& rationale for 10 use. JEMS, 2007;32:S23-S25.

18. Wagner MB, McCabe JB - A comparison of four techniques to establish intraosseous infusion. Pediatr Emerg Care, 1988;4:87-91.

19. Miner WF, Corneli HM, Bolte RG et al - Prehospital use of intraosseous infusion by paramedics. Pediatr Emerg Care, 1989;5:5-7. 
20. 2005 International Consensus on Cardiopulmonary Resuscitation and Emergency Cardiovascular Care Science with Treatment Recommendations. Part 4: Advanced Life Support. Resuscitation, 2005;67:213-247.

21. American Heart Association. Handbook of Emergency Cardiovascular Care for Healthcare Providers. Dallas, American Heart Association, 2006:100.

22. Flato UAP, Flato E, Guimarães HP - Acessos para Administração de Medicamentos, em: Timerman S, Gonzáles MM, Quilici AP - Guia Prático para o ACLS. Barueri: Manole, 2008:132-148.

23. Lopes RD, Falcão LFR, Guimarães HP - Fármacos Rotineiramente Utilizados em Emergências Cardiovasculares, em: Costa MPF, Guimarães HP - Ressuscitação Cardiopulmonar: Uma Abordagem Multidisciplinar. São Paulo: Atheneu, 2007:121-144.

24. Flato UAP, Santos ISO, Ritt LEF - Acessos Venosos em Emergências Clínicas. Em: Lopes AC, Guimarães HP, Lopes RD - PROURGEN - Programa de Atualização em Medicina de Urgência. SBCM. Porto Alegre: Artmed, 2007;127-155.

25. Chameides L - Reanimação Pediátrica, em: Lane JC, Albarran-Sotelo - A Reanimação Cardiorrespiratória Cerebral. Rio de Janeiro, Medsi, 1993;369-416.

26. Nijssen-Jordan C - Emergency department utilization and success rates for intraosseous infusion in pediatric resuscitations. CJEM, 2000;2:10-14

27. Findlay J, Johnson DL, Macnab AJ et al - Paramedic evaluation of adult intraosseous infusion system. Prehosp Disaster Med, 2006;21:329-334.

28. Fowler R, Gallagher JV, Isaacs SM et al - The role of intraosseous vascular access in the out-of-hospital environment (resource document to NAEMSP position statement). Prehosp Emerg Care, 2007;11:63-66.

29. Orlowski JP, Porembka DT, Gallagher JM et al - Comparison study of intraosseous, central intravenous and peripheral intravenous infusions of emergency drugs. Am J Dis Child, 1990;144:112-117.

30. Guy J, Haley K, Zuspan SJ - Use of intravenous infusion in the pediatric trauma patient. J Pediatr Surg, 1993;28:158-161.

31. American Heart Association Guidelines for Cardiopulmonary Resuscitation and Emergency Cardiovascular Care. Pediatric Advanced Life Support American Heart Association. Circulation. 2005;112:(Suppl I::IV167-IV-187.

32. Johnson L, Kissoon N, Fiallos M et al - Use of intraosseous blood to assess blood chemistries and hemoglobin during cardiopulmonary resuscitation with drug infusions. Crit Care Med, 1999;27:1147-1152.

33. Chameides L - Comunicação pessoal. 2007 .

34. Kruse JA, Vyskocil JJ, Haupt MT - Intraosseous infusions: a flexible option for the adult or child with delayed, difficult, or impossible conventional vascular access. Crit Care Med, 1994;22:728-729.
35. Turkel $\mathrm{H}$ - Deaths following sternal puncture. AMA Arch Surg, 1956;73:183-184

36. Dubick MA, Holcomb JB - A review of intraosseous vascular access: current status and military application. Mil Med, 2000;165::552-559.

37. Eisenkraft A, Gilat E, Chapman S et al - Efficacy of the bone injection gun in the treatment of organophosphate poisoning. Biopharm Drug Dispos, 2007;28:145-150.

38. Von Hoff DD, Kuhn JG, Burris HA $3^{\text {rd }}$ et al - Does intraosseous equal intravenous? A pharmacokinetic study. Am J Emerg Med, 2008;26:31-38.

39. McCarthy G, Buss $P$ - The calcaneum as a site for intraosseous infusion. J Accid Emerg Med, 1998;15:421.

40. Macnab A, Christenson J, Findlay $\mathrm{J}$ et al - A new system for sterna intraosseous infusion in adults. Prehosp Emerg Care, 2000;4:173-177.

41. Waisman M, Roffman M, Bursztein S et al - Intraosseous regional anesthesia as an alternative to intravenous regional anesthesia. J Trauma, 1995;39:1153-1156.

42. Iwama $\mathrm{H}$, Katsumi A, Shinohara $\mathrm{K}$ et al - Clavicular approach to intraosseous infusion in adults. Fukushima J Med Sci, 1994;40:1-8.

43. Buck ML, Wiggins BS, Sesler JM - Intraosseous drug administration in children and adults during cardiopulmonary resuscitation. Ann Pharmacother, 2007;41:1679-1686.

44. Miller DD, Guimond G, Hostler DP et al - Feasibility of sternal intraosseous access by emergency medical technician students. Prehosp Emerg Care, 2005;9:73-78.

45. Clem M, Tierney P - Intraosseous infusions via the calcaneus. Resuscitation, 2004;62:107-112.

46. Frascone R, Kaye K, Dries D et al - Successful placement of an adult sternal intraosseous line through burned skin. J Burn Care Rehabil, 2003;24:306-308.

47. LaRocco BG, Wang HE - Intraosseous infusion. Prehosp Emerg Care, 2003;7:280-285.

48. Vojtko M, Hanfling D - The sternal $\mathrm{IO}$ and vascular access-any port in a storm. Air Med J, 2003;22:32-35.

49. Hurren JS - Can blood taken from intraosseous cannulations be used for blood analysis? Burns, 2000;26:727-730.

50. Voelckel WG, Lindner KH, Wenzel V et al - Intraosseous blood gases during hypothermia: correlation with arterial, mixed venous, and sagittal sinus blood. Crit Care Med, 2000;28:2915-2920.

51. Ummenhofer W, Frei FJ, Urwyler A et al - Are laboratory values in bone marrow aspirate predictable for venous blood in pediatrics patients? Resuscitation, 1994;27:123-128.

52. Menezes FH - Acesso à Circulação Venosa, em: Lane JC, Albarran-Sotelo R - Reanimação Cardiorrespiratória Cerebral. Rio de Janeiro: Editora Medsi, 1993;177. 